This is a print version of the following published document:

A. Moreno-Barrado, R. Gago, A. Redondo-Cubero, L. Vázquez, J. MuñozGarcía, R. Cuerno, K. Lorenz, and M. Castro (2015). "Ion damage overrides structural disorder in silicon surface nanopatterning by lowenergy ion beam sputtering" in EPL 109 (2015) 4, 48003.

Available in http://dx.doi.org/10.1209/0295-5075/109/48003

(C) EPLA, 2015 


\title{
Ion damage overrides structural disorder in silicon surface nanopatterning by low-energy ion beam sputtering
}

\author{
A. Moreno-Barrado ${ }^{1,7}$, R. Gago ${ }^{2}$, A. Redondo-Cubero ${ }^{3}$, L. VÁzquez $^{2}$, J. Muñoz-García ${ }^{4,7}$, \\ R. Cuerno ${ }^{4,7}$, K. Lorenz 5 and M. Castro ${ }^{6,1,7}$ \\ 1 Instituto de Investigación Tecnológica (IIT), Universidad Pontificia Comillas - 28015 Madrid, Spain \\ ${ }^{2}$ Instituto de Ciencia de Materiales de Madrid, Consejo Superior de Investigaciones Cientificas \\ 28049 Madrid, Spain \\ 3 Departamento de Física Aplicada y Centro de Microanálisis de Materiales, Universidad Autónoma de Madrid, \\ Cantoblanco - 28049 Madrid, Spain \\ 4 Departamento de Matemáticas, Universidad Carlos III de Madrid - 28911 Leganés, Spain \\ ${ }^{5}$ IPFN, CTN, Instituto Superior Técnico, Universidade de Lisboa - 2695-066 Bobadela LRS, Portugal \\ ${ }^{6}$ Grupo de Dinámica No Lineal (DNL), Escuela Técnica Superior de Ingeniería (ICAI), \\ Universidad Pontificia Comillas - 28015 Madrid, Spain \\ 7 Grupo Interdisciplinar de Sistemas Complejos (GISC)
}

received 15 December 2014; accepted in final form 6 February 2015

published online 25 February 2015

PACS 81.16.Rf - Micro- and nanoscale pattern formation

PACS 68.35.Ct - Interface structure and roughness

PACS 79.20.Rf - Atomic, molecular, and ion beam impact and interactions with surfaces

\begin{abstract}
We investigate the role of the initial structural condition in silicon surface nanopatterning by low-energy ion beam sputtering. Specifically, we address the influence of the target atomic structure in ripple formation under oblique irradiation by $500 \mathrm{eV} \mathrm{Ar}{ }^{+}$ions. To this end, we compare results obtained on single-crystal, amorphous, and pre-implanted silicon targets. In spite of the differences in terms of structural order, and in contrast to previous results for medium energies, surface dynamics are found to be quantitatively similar in all these systems. We explain our results through molecular dynamics simulations of the initial irradiation stages, with the conclusion that the damage induced by low-energy ion bombardment overrides the initial atomic state of the silicon target, irrespectively of its preparation method and allows silicon re-using for nanostructuring.
\end{abstract}

Copyright (C) EPLA, 2015

Surface nanopattern formation by Ion Beam Sputtering (IBS) is a versatile, low-cost technique, applicable to different materials including semiconductors, insulators, and metals $[1,2]$. It allows to produce ordered surface features (e.g., ripples or dots) down to a few $\mathrm{nm}$ in size $[3,4]$. Indeed, IBS-induced surface patterns can be used in several applications, particularly as templates for further nanofabrication [5]. Morphologically, strong similarities occur with e.g. ripple formation on sand dunes [6], as already noted in the first historic reports on IBS surface nanorippling [7]. However, although the technique has been already known for half a century, its underlying mechanisms are still under debate [5]. Hence, from a basic point of view, IBS-induced structures still pose challenging questions on the dynamics of surface patterns at the nanoscale, including issues on pattern order [8], coarsening [9], and kinetic roughening [10].

The case of silicon ( $\mathrm{Si}$ ) targets is focusing a large deal of attention [5], as low-dimensional Si structures like nanowires are currently demonstrating a number of promising, novel structural, electronic, and transport properties $[11,12]$, with potential for a new generation of quantum devices [13]. Actually, recent assessment of the key dynamical role played by unwanted metal incorporation has enabled an improved understanding of experiments with clean, mono-elemental Si targets [14-17]. As a result, ion-induced viscous flow of a shallow surface layer has been identified as an important relaxation mechanism in these systems [18]. Generally, semiconductors become amorphous under ion bombardment [19] but, although 
IBS amorphization of bulk $\mathrm{Si}$ is comparatively well understood [20], the form in which the atomic disorder of the amorphous layer may influence the IBS-induced surface morphology at nanometric scales remains largely unknown. Non-trivial effects in the stress distribution or in the density might be expected, particularly in view of the low ion fluxes employed in IBS surface nanopatterning, roughly 1 ion $\mathrm{nm}^{-2} \mathrm{~s}^{-1}$ or lower [5]. For medium ion energies $(\gtrsim 15 \mathrm{keV})$, oblique $\mathrm{Ar}^{+}$irradiation experiments [21] show attenuated ripple formation for targets in which an initial surface amorphous layer is thicker than the projected ion range. Such a result not only underscores the eventual role of the buried interface separating the iondamaged region from the bulk $[21,22]$ but also suggests that the initial state of the irradiated surface layer can be relevant in the surface nanopatterning. For example, it has been reported [23] that, under $16.7 \mathrm{keV} \mathrm{O}+$ bombardment, increasing the initial surface roughness of silicon targets can enhance and amplify pattern formation. However, at low ion energies $(\lesssim 1 \mathrm{keV})$ and without the impact of metal impurities, as in, e.g., [14-18], it is not known whether the initial state of the Si target in terms of atomic disorder might influence ripple formation. This case is particularly important due to the widespread use of affordable and versatile low-energy IBS equipments for surface nanopatterning.

In this letter we show that it is not the initial atomic disorder but, rather, the (additional) ion-induced damaging within a shallow surface amorphous layer, which controls the onset and dynamics of patterns for low-energy IBS of Si. To this end, we have studied nanoripple formation under $500 \mathrm{eV} \mathrm{Ar}^{+}$oblique irradiation in the case of four different initial structural conditions. Surface analysis has been performed through ex situ Atomic Force Microscopy (AFM) and is complemented with Molecular Dynamics (MD) simulations to determine the local disorder before and after low-energy IBS, for spatial and temporal scales that are experimentally inaccessible. Our experimental results show quantitatively that under the present sputtering conditions all the systems display the same surface dynamics, despite structural differences in the initial conditions. This is explained by the MD simulations, which lead to the conclusion that the ion-induced damage overrides the initial state of the targets, irrespectively of their structural properties, by inducing the same type of viscous flow for the damaged surface layer. In turn, it is this viscous flow which controls the ripple formation process at the surface.

The systems used in this work are summarized in table 1. First, a $380 \mu \mathrm{m}$ thick single-crystalline $\mathrm{Si}(100)$ target (c-Si) was employed as a reference ordered system. Second, $\mathrm{a} \simeq 1.2 \mu \mathrm{m}$ thick silicon film grown on $\mathrm{Si}(100)$ was taken as a reference amorphous $\mathrm{Si}(\mathrm{a}-\mathrm{Si})$. Growth was performed by DC magnetron sputtering at room temperature in a pure Ar atmosphere from a high-purity $\mathrm{Si}(99.95 \%)$ sputter target at a power of $25 \mathrm{~W}$ (see ref. [24] for further details). Finally, two Si(100) wafers were implanted (i-Si)
Table 1: Summary of the Si systems used in this work. The initial disordered thickness refers to the layer thickness of the as-grown amorphous film or the damaged one created by ion pre-implantation.

\begin{tabular}{lcl}
\hline \hline Sample description & Label & $\begin{array}{l}\text { Initial } \\
\text { disordered } \\
\text { thickness }\end{array}$ \\
\hline Pristine Si(100) wafer & $\mathrm{c}-\mathrm{Si}$ & - \\
Amorphous Si grown film & $\mathrm{a}-\mathrm{Si}$ & $\simeq 1.2 \mu \mathrm{m}$ \\
$200 \mathrm{keV} \mathrm{Ar}^{+}$implanted $\mathrm{Si}$ & $\mathrm{i}-\mathrm{Si}(\mathrm{Ar})$ & $\simeq 330 \mathrm{~nm}$ \\
$150 \mathrm{keV} \mathrm{Si}^{+}$implanted $\mathrm{Si}$ & $\mathrm{i}-\mathrm{Si}(\mathrm{Si})$ & $\simeq 240 \mathrm{~nm}$ \\
\hline \hline
\end{tabular}

to produce pre-amorphization of the outer layers. We employed $200 \mathrm{keV} \mathrm{Ar}^{+}$and $150 \mathrm{keV} \mathrm{Si}^{+}$ions, respectively, in order to achieve damaged layers of a similar ion projected range (around $200 \mathrm{~nm}$, based on TRIM simulations [25]). The self-implanted sample was used as a control since Ar implantation sometimes leads to the local formation of gas bubbles. However, this effect was also minimized by implanting both samples with a fluence of $5 \times 10^{15}$ ions $/ \mathrm{cm}^{2}$, low enough to avoid eventual microstructural features. All these different conditions were chosen because several physical properties of amorphous Si are known to depend on the preparation method. E.g., a-Si prepared by physical vapor deposition can show a density deficit relative to c-Si larger than 10\%, while the density of a-Si prepared by ion implantation is only around $2 \%$ less than that of c-Si [20]. The four systems we study have a small initial surface roughness $(\simeq 0.2 \mathrm{~nm})$, which should not play a role in the dynamics. The non-crystalline character of the a-Si and i-Si samples has been verified by spectroscopic ellipsometry (SE), also used to determine the (damaged) layer thickness, see table 1. The combination of SE and Rutherford Backscattering Spectrometry (RBS) also allowed to verify the density values of the as-preperated targets, according to ref. [26].

The four systems were subsequently subjected to $500 \mathrm{eV}$ $\mathrm{Ar}^{+}$IBS to induce surface nanoripples, as in ref. [18]. The samples were attached to a sacrificial Si wafer and the absence of (metal) impurities after irradiation was checked by RBS. The beam was extracted from a $3 \mathrm{~cm}$ beam-diameter Kaufman-type ion gun impinging at $65^{\circ}$ with respect to the target normal. The current density at the sample position (parallel to the ion beam) was set to $50 \mu \mathrm{A} / \mathrm{cm}^{2}$. The erosion rate was always $\simeq 3.1 \mathrm{~nm} / \mathrm{min}$, as assessed by SE. Surface topographies were imaged ex situ with Nanoscope IIIa (Bruker) and Agilent 5500 (Agilent) AFM equipments in intermittent-contact mode, using Si cantilevers with $8 \mathrm{~nm}$ nominal curvature radius.

Figures 1(a)-(d) show the AFM images obtained after 20 minutes of irradiation for the systems in table 1 . Beyond visual similarities, fig. 1(e) depicts a similar power spectral density (PSD) for all four systems, revealing the independence of the pattern morphology with respect to 

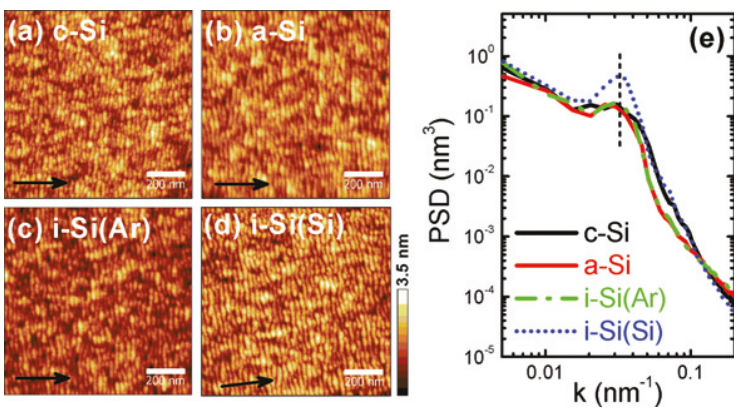

Fig. 1: (Colour on-line) (a)-(d) AFM images of nanoripples produced by IBS after 20 minutes of irradiation for the four different targets in table 1 . The arrows show the direction of the $\mathrm{Ar}^{+}$beam. (e) Corresponding PSD of the morphologies.

the initial state of the target. Figure 2 displays the evolution of the ripple wavelength $\ell$ (fig. 2(a)) and roughness $w$ (fig. 2(b)). Remarkably, the four systems display the same dynamics quantitatively (within statistical deviations), despite their different initial atomic structures. Actually, for the i-Si(Ar) sample the amorphous-crystalline interface generated by pre-implantation has been reached after $100 \mathrm{~min}$ (see arrow in fig. 2), without any noticeable effect in the dynamics. This is at variance with results at medium energy [21]. Here, the authors found that the onset for pattern formation occurs later for an initial thicker amorphous layer although, once the instability emerges, the dynamics of the pattern wavelength is rather similar to the thinner case. In contrast, the ripple amplitude increases (remains constant and much smaller) with irradiation time for an initial thin (thick) amorphous layer. As already mentioned, this behavior highlights the role of the amorphous layer and the need of medium-energy ions reaching the underlying crystalline interface to effectively induce a pattern [21]. As our present results indicate, the situation may differ at low-energy irradiations. Also, under metal co-deposition equivalent dynamics have also been reported for nanodot patterns produced on amorphous and crystalline Si targets [24].

With the aim of determining the causes behind this loss of memory of the initial state of the material after low-energy IBS, we have investigated the microscopic properties of the four systems before and after low-energy irradiation by means of MD simulations, performed using the LAMMPS software [27]. The interaction between particles was modeled by Tersoff potential [28] interpolated with a Ziegler-Biersack-Littmark [25] term. This choice allows to accurately account for the elastic relaxation and therefore a better description of amorphous Si [29]. The size of the systems was $4.9 \times 4.9 \times 10.9 \mathrm{~nm}$. A Si crystal with a diamond cubic structure equilibrated to $300 \mathrm{~K}$ was employed to simulate the c-Si sample. Two different approaches were used for the amorphous/implanted systems. On the one hand, a melted and quenched c-Si target obtained following the method described in refs. [30,31] was generated as a reference for the a-Si layer. On the other hand, to reproduce the i-Si targets, the as-generated

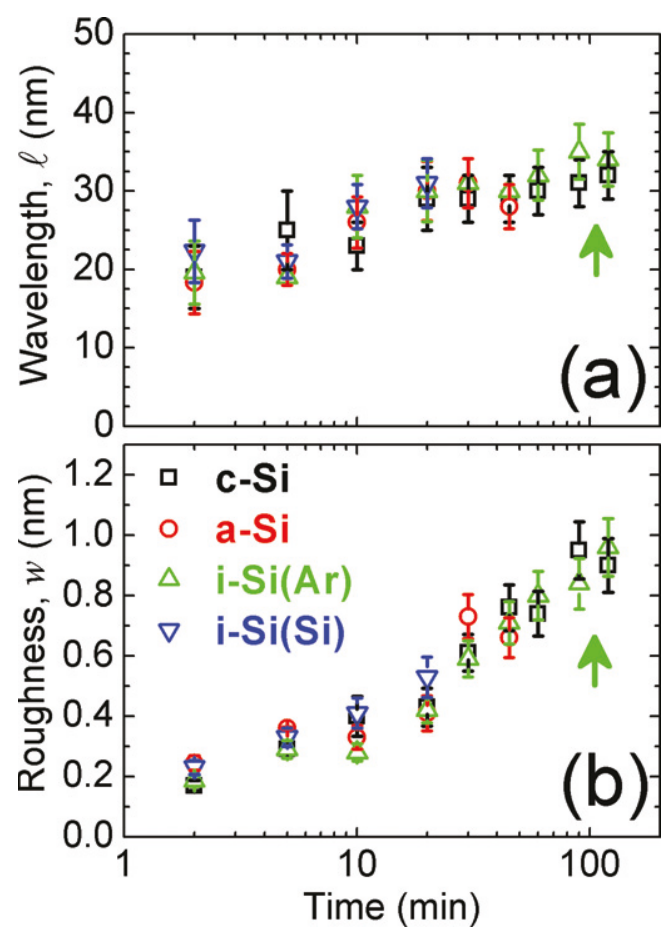

Fig. 2: (Colour on-line) Temporal evolution of the pattern wavelength (a) and roughness (b) for the different initial $\mathrm{Si}$ targets in table 1 . The vertical green arrow indicates the irradiation time for which the interface between the pre-implanted layer and the underlying crystalline silicon bulk has been reached for the i-Si set (note that there is no noticeable effect in the dynamics when this point is gone through).

c-Si structure was subsequently subjected to $150 \mathrm{keV} \mathrm{Si}{ }^{+}$ or $200 \mathrm{keV} \mathrm{Ar}^{+}$irradiation for a fluence value like the one used in the experiments. A final irradiation with $500 \mathrm{eV} \mathrm{Ar}^{+}$ions was then carried out on the four generated structures, for a total of 1000 consecutive impacts. This contrasts with most standard MD approaches, in which averages are performed over different impacts on the same pristine material. Since the collision cascade is around 2unit-cells wide $(\simeq 1 \mathrm{~nm})$, the system size is large enough so as to prevent any influence of the boundaries, where the thermostats are located. In ref. [32], an equivalence between near-normal and grazing incidence conditions has been established for the bulk damage. Namely, the stress distribution under oblique incidence follows from that at a different condition (e.g. normal incidence) through a simple tensor rotation. Here, we exploit this result and perform simulations at $5^{\circ}$ to normal, to avoid channeling effects. Further simulation details are as in [32].

In order to quantify the structural atomic order in the simulations, the density, the radial distribution function, $g(r)$, and the coordination number are shown in fig. 3 . Figures 3(a) and (b) show the density relative to that of the crystal for the four systems in table 1 before and after irradiation, respectively. Both c-Si and a-Si have similar densities within error bars, while the implanted samples just show a slight atomic deficit, in agreement with the 

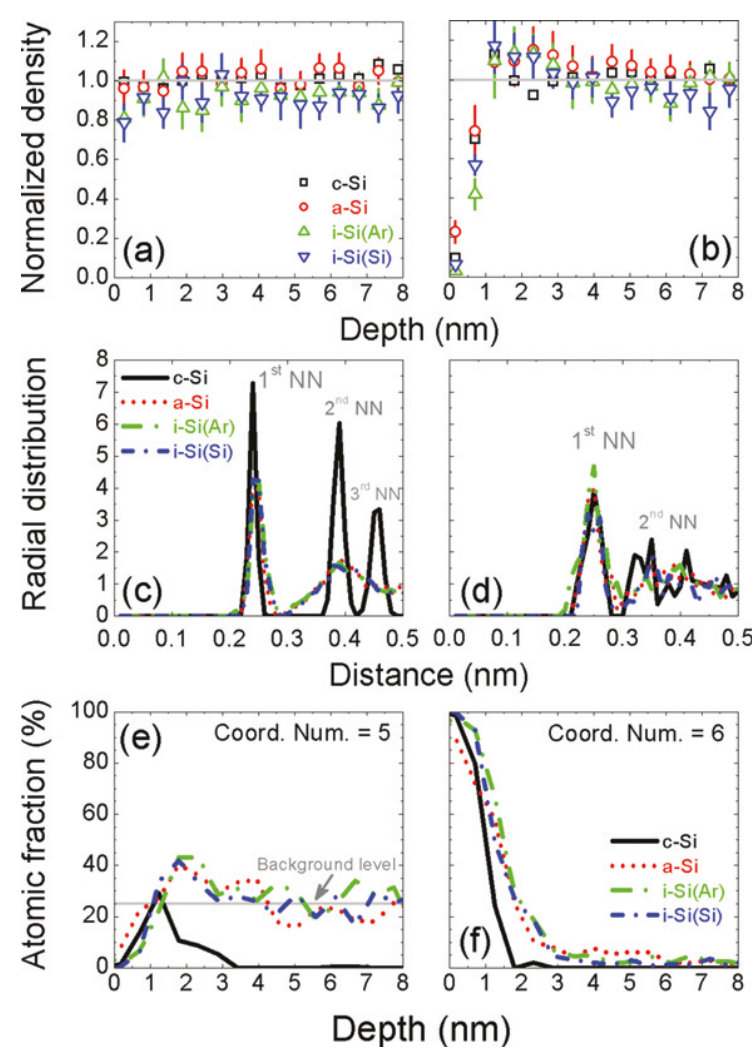

Fig. 3: (Colour on-line) Normalized atomic density ((a) and (b)) and radial distribution function ((c) and (d)) before and after $1000 \mathrm{Ar}^{+}$impacts, respectively. Atomic fraction of $\mathrm{Si}$ atoms with coordination number equal to 5 (e) and 6 (f) after $1000 \mathrm{Ar}^{+}$impacts.

experimental SE observations. After IBS, the Si density drops noticeably at the surface, concurrent with a densification near the end-of-range region.

Figures 3(c) and (d) show the radial distribution function, defined as

$$
g(r)=\frac{1}{\rho}\left\langle\sum_{i \neq 0} \delta\left(\mathbf{r}-\mathbf{r}_{i}\right)\right\rangle=V \frac{N-1}{N}\left\langle\delta\left(\mathbf{r}-\mathbf{r}_{i}\right)\right\rangle,
$$

where $\rho$ is density, $\mathbf{r}_{i}$ are the neighbor positions within a distance of $0.5 \mathrm{~nm}$ from a particle considered at $\mathbf{r}$, $N$ is their number, $V$ is volume, and the brackets denote particle average. This function contains information about the average distance between atoms. Initially, see fig. 3(c), a-Si and i-Si exhibit an identical functional shape, with two main broad peaks reflecting short-range order. Only c-Si displays narrow peaks and long-range order (not shown). Nevertheless, after irradiation (fig. 3(d)) the radial distribution function, measured only in the top damaged layer, is similar in all the systems.

The coordination number takes into account the number of first Nearest Neighbors (NN) surrounding each Si atom. We consider the neighbors within a distance of $3 \mathrm{~nm}$, which is higher than the radial distance of the first-NN and lower than second-NN distance of diamond-cubic Si. Figures 3(e) and (f) show the fraction of Si atoms after irradiation which have coordination number equal to 5 and 6, respectively. The remarkable increase in 5-fold and 6 -fold coordination with respect to as-prepared samples (which feature a low background of 5-fold coordinated atoms and the complete absence of 6 -fold coordination), indicates the formation of interstitials in the damaged surface layer. Interestingly within this layer, roughly $2 \mathrm{~nm}$ wide, all the systems have identical properties.

These results indicate that, through irradiation, similar structural disorder ensues in the topmost surface layer for all the targets. Thus, the rearrangements induced by sustained IBS eventually govern its atomic structure, independently of its previous history. Within the ioninduced viscous flow theory for low-energy IBS $[18,32,33]$, ripple formation is controlled by the relaxation of such a damaged surface layer, once it is formed. This is driven by the large ion-induced residual stress which occurs for the present range of energies, as predicted by Davis' model $[18,26]$. According to the theory in refs. [18,32,33], the independence of the atomic structure in the damaged layer with respect to its previous state leads to a similar morphological dynamics — as assessed by the roughness and the ripple wavelength - for all the targets considered, as we obtain experimentally here. Other theoretical descriptions, like the classic Bradley-Harper (BH) model [34], neglect both the structural changes within the substrate and the evolution of the damaged layer. Refinements of the BH theory, known as crater-function methods [29,35-37], perform averages over the matter rearrangement events induced by single ion impacts, in order to evaluate the evolution of the surface [38], but similarly neglect the structural atomic damage created within the target.

In conclusion, we provide experimental and theoretical evidences that support the irrelevance of the initial structural state of silicon targets for surface nanopatterning under low-energy, impurity-free IBS conditions. This contrasts with previous results obtained at medium energies [21], questioning the straightforward relation between both energy conditions that is frequently hypothesized in the literature [5].

Our present mesoscopic analysis through AFM measurements and MD simulations both support the interpretation that the damage created by the irradiation overrides the atomic structure -irrespective of its crystallinity or of the origin of the pre-amorphized layer - in the topmost surface layer which controls ripple formation. While supporting the relevance of relaxation by ion-induced viscous flow as the basic mechanism controlling pattern formation in the present class of systems, this result has the important practical consequence that the characteristics and quality of the nanopatterns thus produced do not depend on the previous structural state of the material. This conclusion suggests that previously irradiated silicon targets could be re-used in new nanopatterning experiments; as shown in this work, all the existing structure will be overridden by the secondary low-energy irradiation. 
$* * *$

This work has been partially supported by MICINN (Spain) grant MAT2011-13333-E, MINECO (Spain) grants FIS2012-38866-C05-01, FIS2012-38866-C05-05, FIS2012-32349, FIS2013-47949-C2-2-P, and Comunidad Autónoma de Madrid grant NANOAVANSENS S2013/MIT-3029. AM-B and AR-C acknowledge support from MINECO (SPAIN) through FPI scolarship BES-2010-036179 and Juan de la Cierva contract No. JCI-2012-14509, respectively.

\section{REFERENCES}

[1] Chan W. L. and Chason E., J. Appl. Phys., 101 (2007) 121301.

[2] Muñoz-García J., Vázquez L., Cuerno R., SánchezGarcía J. A., Castro M. and Gago R., Self-organized surface nanopatterning by ion beam sputtering, in Toward Functional Nanomaterials (Springer) 2009, pp. 323-398.

[3] Facsko S., Dekorsy T., Koerdt C., Trappe C., Kurz H., Vogt A. and Hartnagel H. L., Science, 285 (1999) 1551.

[4] Wei Q., Lian J., Zhu S., Li W., Sun K. and Wang L., Chem. Phys. Lett., 452 (2008) 124.

[5] Muñoz-García J., Vázquez L., Castro M., Gago R., Redondo-Cubero A., Moreno-Barrado A. and Cuerno R., Mater. Sci. Eng.: R: Rep., 86 (2014) 1.

[6] Aste T. and Valbusa U., New J. Phys., 7 (2005) 122.

[7] Navez M., Sella C. and Chaperot D., C. R. Acad. Sci., 254 (1962) 240.

[8] Bradley R. M. and Shipman P. D., Phys. Rev. Lett., 105 (2010) 145501.

[9] Muñoz-García J., Gago R., Vázquez L., SánchezGarcía J. A. and Cuerno R., Phys. Rev. Lett., 104 (2010) 026101.

[10] Vivo E., Nicoli M., Engler M., Michely T., Vázquez L. and Cuerno R., Phys. Rev. B, 86 (2012) 245427.

[11] Ou X., Kögler R., Wei X., Mǘchlich A., Wang X., Skorupa W. and Facsko S., AIP Adv., 1 (2011) 042174.

[12] Rurali R., Rev. Mod. Phys., 82 (2010) 427.

[13] Zwanenburg F. A., Dzurak A. S., Morello A., Simmons M. Y., Hollenberg L. C. L., Klimeck G., Rogge S., Coppersmith S. N. and Eriksson M. A., Rev. Mod. Phys., 85 (2013) 961.

[14] Madi C., Davidovitch B., George H., Norris S., Brenner M. and Aziz M., Phys. Rev. Lett., 101 (2008) 246102.
[15] Madi C., Anzenberg E., Ludwig K. jr. and Aziz M., Phys. Rev. Lett., 106 (2011) 066101.

[16] Hofsäss H., Zhang K., Gehrke H. and Brüsewitz C., Phys. Rev. B, 88 (2013) 075426.

[17] Engler M., Macko S., Frost F. and Michely T., Phys. Rev. B, 89 (2014) 245412.

[18] Castro M., Gago R., Vázquez L., Muñoz-García J. and Cuerno R., Phys. Rev. B, 86 (2012) 214107.

[19] Gnaser H., Low Energy Ion Irradiation of Solid Surfaces (Springer-Verlag) 1999.

[20] Pelaz L., Marqués L. A. and Barbolla J., J. Appl. Phys., 96 (2004) 5947.

[21] Kumar T., Kumar A., Agarwal D. C., Lalla N. P. and Kanjilal D., Nanoscale Res. Lett., 8 (2013) 336.

[22] Kumar T., Kumar A. and Kanjilal D., Appl. Phys. Lett., 103 (2013) 131604.

[23] Karmakar P., Mollick S., Ghose D. and Chakrabarti A., Appl. Phys. Lett., 93 (2008) 103102.

[24] Muñoz-García J., Gago R., Cuerno R., SánchezGarcía J., Redondo-Cubero A., Castro M. and VÁzquez L., J. Phys.: Condens. Matter, 24 (2012) 375302 .

[25] Ziegler J. F., Biersack J. P. and Littmark U., The Stopping and Range of Ions in Matter (Pergamon, New York) 1985.

[26] Davis C. A., Thin Solid Films, 226 (1993) 30.

[27] Plimpton S., J. Comput. Phys., 117 (1995) 1.

[28] Tersoff J., Phys. Rev. B, 38 (1988) 9902.

[29] Norris S. A., Samela J., Bukonte L., Backman M., Djurabekova F., Nordlund K., Madi C. S., Brenner M. P. and Aziz M. J., Nat. Commun., 2 (2011) 276.

[30] Chokappa D. K. and Clancy P., Mol. Phys., 61 (1987) 597.

[31] Chokappa D. K. and Clancy P., Mol. Phys., 61 (1987) 617.

[32] Moreno-Barrado A., Castro M., Gago R., Vázquez L., Muñoz-García J., Redondo-Cubero A., Galiana B., Ballesteros C. and Cuerno R., submitted to Phys. Rev. B (2014).

[33] Castro M. and Cuerno R., Appl. Surf. Sci., 258 (2012) 4171.

[34] Bradley R. M. and Harper J. M. E., J. Vac. Sci. Technol. A, 6 (1988) 2390.

[35] Hossain M. Z., Das K., Freund J. B. and Johnson H. T., Appl. Phys. Lett., 99 (2011) 151913.

[36] Nietiadi M. L. and Urbassek H. M., Appl. Phys. Lett., 103 (2013) 113108.

[37] Harrison M. P. and Bradley R. M., Phys. Rev. B, 89 (2014) 245401.

[38] Norris S. A., Brenner M. P. and Aziz M. J., J. Phys.: Condens. Matter, 21 (2009) 224017. 